\title{
A perspective on experimental findings and theoretical explanations of novel dynamics at free surface and in freestanding thin films of polystyrene
}

\author{
K. L. Ngai ${ }^{\mathrm{a} *}$, Daniele Prevosto ${ }^{\mathrm{a}}$, S. Capaccioli ${ }^{\mathrm{a}, \mathrm{b}}$ \\ ${ }^{a}$ CNR-IPCF, Largo B. Pontecorvo 3, I-56127, Pisa, Italy \\ ${ }^{b}$ Dipartimento di Fisica, Università di Pisa, Largo B. Pontecorvo 3, I-56127, Pisa, Italy
}

\begin{abstract}
Most studies of the dynamics at the surface and in thin films of polystyrene (PS) are focused on the change of glass transition temperature from the bulk value. In this perspective we highlight three new developments in research on the dynamics of PS in high molecular weight (MW) freestanding PS thin films and at the surface of low MW PS. Novel findings from these developments require consideration of viscoelastic mechanisms with length-scales longer than the segmental $\alpha$-relaxation. The first development is the creep compliance measurements of high MW PS thin films, probing not only the segmental $\alpha$-relaxation but also the polymer chain modes at higher compliance levels, including the sub-Rouse modes and the Rouse modes. The compliance data indicate the relaxation times of the sub-Rouse modes are reduced in thinner films like that of the segmental $\alpha$-relaxation but to a much less extent. The second development is the novel observation of two glass transitions in freestanding polystyrene thin films by ellipsometry. The upper and lower glass transitions occurs respectively at temperatures $T_{g}^{u}$ and $T_{g}^{l}$, both are below the bulk glass transition temperature. While the lower transition at $T_{g}^{l}$ is associated with the segmental $\alpha$-relaxation, the only viable explanation of the origin of the upper transition at $T_{g}^{u}$ is from another intrinsic viscoelastic
\end{abstract}


mechanism of PS, and not at different location of the film. Supported by various experiments on PS and other polymers, we show that the sub-Rouse modes are cooperative and coupled to density, and hence giving rise to the upper glass transition in freestanding PS films. The subRouse relaxation times will increase on physical aging, and bring along an increase in density of the freestanding film due to the coupling. This prediction can be checked by performing aging experiment. The third development is the reduction of viscosity at the free surface of low MW PS. Since viscous flow of low MW PS is definitely carried out by the sub-Rouse modes and not by the segmental $\alpha$-relaxation, the experimental finding is direct evidence of enhancement of mobility of the sub-Rouse modes by the mitigation of intermolecular coupling at the surface, consistent with the explanations given for the findings in the two other developments. The enhancement of mobility of the sub-Rouse modes occur simultaneously with the same effect on the segmental $\alpha$-relaxation. Notwithstanding, at the surface, the observed reduction of viscosity from the sub-Rouse modes is significantly smaller than the reduction of the segmental relaxation time, which is explained semi-quantitatively. Altogether, the three recent advances in the study of dynamics of polymer thin films and at the free surface have shown not only the change of the glass transition temperature effected by the segmental $\alpha$-relaxation is interesting, but also that of the sub-Rouse modes.

*Corresponding author. Email: kia.ngai@pi.ipcf.cnr.it 


\section{Introduction}

Recently published are three novel and important developments in the experimental studies of reductions of glass transition temperature $T_{g}$ in high molecular weight freestanding polymer films [1-3], and the reduction of viscosity at the surface of low molecuar weight polymer $[4,5]$. All three developments have impact on the frontier of research in the field.

(1) One development is the viscoelastic biaxial creep compliance, $D(t)$, measurements of ultra-thin films of high molecular weight polystyrene (PS) [1], and polycarbonate (PC) [2] by McKenna and co-workers. A large reduction of $T_{g}$ on decreasing film thickness $h$ was observed. However, observed also is the anomalous or surprising decrease of the rubbery plateau compliance on decreasing $h$. The implication of the effect is that the viscoelastic mechanisms (sub-Rouse modes and Rouse modes to be specify later) contributing to higher compliances than the segmental $\alpha$-relaxation are not enhanced in mobility as much as the $\alpha$ relaxation with the decrease of $h$. [6]

(2) Pye and Roth [3] presented evidence from ellipsometry measurements of the presence of two glass transitions originating from two distinctly different and simultaneous mechanisms to reduce the glass transition temperature within freestanding high molecular weight polystyrene (PS) films with thickness less than $70 \mathrm{~nm}$. The lower transition at temperature $T_{g}^{l}(h)$ is the same as found before in freestanding PS films by others [7-10], and can be explained by the large enhancement of mobility of the segmental $\alpha$-relaxation due to the free surfaces, and finite size effects including induced orientations of the PS chains [1113]. The upper transition at a higher temperature $T_{g}^{u}(h)$ is novel. The two transitions cannot be explained simply by effects at two different locations of the film. This is because this hypothesis implies the difference, $T_{g}^{u}(h)-T_{g}^{l}(h)$, would decrease as film thickness decreases, 
which is opposite to the increase difference observed experimentally. Therefore, the upper transition must come from another viscoelastic mechanism other than the segmental $\alpha$ relaxation of PS. The sub-Rouse modes have been suggested to give rise to the upper transition [14].

(3) Enhanced viscous flow at the surface of low molecular weight polystyrene with $M_{\mathrm{w}}=3000 \mathrm{~g} / \mathrm{mol}$ was found by Chai et al.[4], confirming the results obtained earlier deduced by Yang et al. [5]. The viscosity at the surface was found smaller than the bulk value, but the reduction is much smaller than that of the segmental $\alpha$-relaxation time at the surface. It is important to recognize that for the low molecular weight polystyrene studied, viscous flow at the surface or in the bulk is carried out not by the segmental $\alpha$-relaxation but by the subRouse modes [6]. Therefore an explanation of the enhanced flow at the surface, and the answer to why it is much less than reduction of segmental $\alpha$-relaxation time at the surface, must come directly from the sub-Rouse modes. However, Chai et al. and Yang et al. made no connection of their surface viscosity data to the sub-Rouse modes, and so far this problem has not been addressed.

All the three new experimental findings are important and crux to a full understanding of the enhancement of mobility at the surface and in freestanding thin films not only the segmental $\alpha$-relaxation (i.e. reduction of $T_{g}$ ) but also the sub-Rouse modes. This task requires first a knowledge of the viscoelasic mechanisms of bulk PS beyond the segmental $\alpha$ relaxation before explaining how the different viscoelastic mechanisms are enhanced in mobility at the surface and in freestanding thin films. This Perspective is written to draw attention of the research community to these fundamentally important experimental findings 
that have impact on dynamics of polymers at the surface and in nano-confinement, and the current level of theoretical understanding.

We give a brief summary the biaxial creep compliance data of PS thin film of McKenna and coworkers $[1,2]$ mentioned in (1). This brings out the novel findings that the enhancement in mobility of the sub-Rouse modes and the Rouse modes is much less than the segmental $\alpha$ relaxation on thinning the PS film [6]. Shear creep compliance data of bulk PS by Plazek and coworkers [15-18] and others [19-21] are invoked to support the identification of sub-Rouse modes and Rouse modes in thin films and the explanation given before [22]. Theoretical predictions from Guenza [23], and neutron scattering experimental data [24] are cited for the same reason and purpose.

Having established the presence of the sub-Rouse modes in PS thin films from (1), next we give a short account of the experimental findings of two glass transitions in freestanding PS films [3], and the interpretation of the upper transition as originating from the sub-Rouse modes [6]. In this paper we give results from the studies at elevated pressure and temperatures to show the sub-Rouse modes is coupled to density and can give rise to the upper glass transition. Also from the coupling to density, we can immediately infer that the sub-Rouse relaxation time $\tau_{s R}$ will shift to longer times like $\tau_{\alpha}$ as the film is densified with time by physical aging. The prediction can be observed in ellipsometry measurements of the freestanding PS film by a decrease of the upper glass transition temperature $T_{g}^{u}(h)$. This new prediction can be checked with physical aging experiment of freestanding PS films when results become known in the literature.

Finally we address the item (3) by providing entirely new and quantitative analysis of the experimental data of viscosity of low molecular weight PS at the surface $[4,5]$. Viscous 
flow in the low molecular weight PS studied is carried out by the sub-Rouse modes, and not by the segmental $\alpha$-relaxation. Thus our analysis and explanation of the experimental data from the enhancement of mobility of the sub-Rouse modes at the surface fill the void of theoretical understanding of viscous flow at the free surface of PS.

As far as we know, no theory or model has been proposed to explain the findings in the new developments (1) and (2) except that from Refs.[6] and [14] by using the Coupling Model (CM) [11-14, 22, 25]. Here in this paper, the CM shall be used once more to interpret and explain the data of the development (3). It is also the only model [26] dealing with all the viscoelastic mechanisms of polymers including the sub-Rouse modes in the bulk [14]. Thus a short summary of the $\mathrm{CM}$ is given first in the next Section before considering the experimental data.

\section{Brief Summary of the CM}

The key equation of the $\mathrm{CM}$ is the dependence of the segmental $\alpha$-relaxation time $\tau_{\alpha}$ on the coupling parameter $n_{\alpha}$ given by

$$
\tau_{\alpha}(T)=\left[\left(t_{c}\right)^{-n_{\alpha}} \tau_{0}(T)\right]^{1 /\left(1-n_{\alpha}\right)}
$$

where $t_{c}=1$ to $2 \mathrm{ps}$ for PS and similar polymers [26] and $\tau_{0}$ is the primitive relaxation time. When Eq.(1) is applied to thin films and free surface, the value of $\tau_{0}$ is the same as in the bulk, i.e. independent of the thickness $h$ and the location of any layer $j$ within the film. Based on Eq.(1), the 2006 paper [13] gives a layer-by-layer description of the attenuation of the free surface effect in thin films. The largest reduction of $n_{\alpha}$ occurs at the free surface. On going towards the interior of the film starting from the surface (i.e. on increasing $j$ ), the reduction of $n_{\alpha}(h, j)$ is attenuated monotonically. It can be easily verified by Eq.(1), the smaller values of 
$n_{\alpha}(h, j)$ than $n_{\alpha, b u l k}$ leads to shorter $\tau_{\alpha}(h, j, T)$ than $\tau_{\alpha, b u l k}(T)$, and correspondingly the overall reduction of the glass transition temperature $T_{g}(h)$ with decrease of $h$, found in supported [27] and freestanding PS thin films [3,7-10].

Experiments in bulk polymers have shown that the sub-Rouse modes are also cooperative but to a lower degree than the segmental $\alpha$-relaxation [17,22,28-33], and have smaller bulk coupling parameter, $n_{s R}$, than $n_{\alpha}$ of the segmental $\alpha$-relaxation. Like the segmental $\alpha$-relaxation, the sub-Rouse modes are also intermolecularly cooperative, albeit to the lesser degree and with a smaller coupling parameter $n_{s R, b u l k}$ than $n_{\alpha, b u l k}$. At the surface, intermolecular coupling and cooperativity of the sub-Rouse modes are reduced for the same reason given for the segmental $\alpha$-relaxation [11-14]. Correspondingly, the coupling parameter of the sub-Rouse modes at the surface is much reduced from the bulk value $n_{s R, b u l k}$. The CM Eq. (1) is general and applicable to all cooperative processes including the sub-Rouse modes in the bulk, at the surface, and in thin film, which takes the form

$$
\tau_{s R}(T)=\left[\left(t_{c}\right)^{-n_{S R}} \tau_{0, s R}(T)\right]^{1 /\left(1-n_{S R}\right)}
$$

where $\tau_{0, \mathrm{sR}}$ is the primitive sR-relaxation time and $t_{c}$ is the same as in Eq.(1). For the same reason, the effects of the free surface and the finite size of thin film cause a reduction of the bulk coupling parameter $n_{s R, b u l k}$ to smaller values of $n_{S R}(h, j)$, where $j$ labels the layers from the surface. In analogy to $\tau_{0}$, also $\tau_{0, \mathrm{SR}}$ is independent of $h$ and $j$. From this result, and by applying Eq.(2) to both the bulk and the thin film, it can be easily verified that $\tau_{S R}(h, j)$ is shorter than $\tau_{s R, b u l k}$. Naturally the smallest value of $n_{s R}(h, j)$ and the shortest $\tau_{s R}(h, j)$ calculated by Eq.(2) are at the $j=1$ layer, i.e. the layer at the free surface.

\section{Evidence of enhancement of mobility of sub-Rouse modes in PS thin films}


Isothermal biaxial creep compliance, $D(t)$, of unsupported nanobubble inflated ultra-thin films of high MW polymers was measured over the glass-rubber transition zone by McKenna and coworkers [1,2]. For film of any thickness $h$, the creep curve shifts to shorter times on decreasing temperature. However, accompanying the shift is the decrease of the rubbery plateau compliance. The decrease becomes more dramatic in thinner films and at lower temperatures. An example of this anomalous behavior of creep compliance taken at 69, 72, and $75{ }^{\circ} \mathrm{C}$ can be seen in Fig. 1 for a $36 \mathrm{~nm}$ thick PS film with MW=994,000 Da. The ranges of the additive contributions to shear compliance from the segmental $\alpha$-relaxation, $\hat{J}_{\alpha}(t)$, the sub-Rouse modes, $\hat{J}_{S R}(t)$, and the Rouse modes, $\hat{J}_{R}(t)$ of PS have been determined by experiments [6]. For entangled high molecular weight PS, it has been shown that $\hat{J}_{\alpha}(t)$ lies within the range bounded by the glassy compliance $J_{g}=0.93 \times 10^{-9} \mathrm{~Pa}^{-1}$ and $J_{e \alpha} \approx 4 \times$ $10^{-9} \mathrm{~Pa}^{-1}$, i.e.,
\[ J_{g} \leq \hat{J}_{\alpha}(t) \leq J_{e \alpha} . \]

The sub-Rouse modes contribution, $\hat{J}_{S R}(t)$, exist within the bounds

$$
J_{e \alpha} \leq \hat{J}_{s R}(t) \leq J_{S R} \approx 10^{-7} \mathrm{~Pa}^{-1} .
$$

The Rouse modes contribution, $\hat{J}_{R}(t)$, fall within the range,

$$
J_{S R} \leq \hat{J}_{R}(t) \leq J_{\text {plateau }}
$$

where $J_{\text {plateau }} \approx 10^{-6} \mathrm{~Pa}^{-1}$ is the entanglement plateau compliance of PS.

From the relation, $J(t)=6 D(t),[1,2,6]$ the sub-Rouse modes contributions, $\widehat{D}_{S R}(t)$, to the biaxial compliance lie within the range,

$$
\left(6.7 \times 10^{-10} \mathrm{~Pa}^{-1} \approx D_{e \alpha}\right) \leq \widehat{D}_{s R}(t) \leq\left(D_{s R} \approx 1.67 \times 10^{-8} \mathrm{~Pa}^{-1}\right) .
$$

The tip and the end of the arrow indicate $D_{s R}$ and $D_{e \alpha}$ respectively. The sole purpose of Fig.1 is to demonstrate the simultaneous observation of the sub-Rouse modes and the segmental $\alpha$ - 
relaxation in thin PS films. By considering the change of the biaxial compliance data on decreasing film thickness $h$, we have explained and concluded in [13] that the mobility of both the sub-Rouse modes and the segmental $\alpha$-relaxation are enhanced on decreasing $h$, but to a lesser extent for the former than the latter. Here we can use Fig.1 to elucidate simply this fact. It is clear from Fig. 1 that the creep compliance $D(t)$ data obtained at 72 and $75{ }^{\circ} \mathrm{C}$ are contributed entirely by the sub-Rouse modes. In order for the sub-Rouse modes of the bulk PS with $\mathrm{MW}=994,000 \mathrm{Da}$ to be seen in the experimental time window of Fig.1, the temperature has to be much higher than $T_{g}=98.8 \mathrm{C}$. Since the sub-Rouse modes appear within the experimental time window at 72 and $75 \mathrm{C}$, therefore the sub-Rouse modes have been shifted to shorter times by the presence of the free surfaces, and possibly also the finite size effect in the $36 \mathrm{~nm}$ thick high-MW PS film.

In Fig. 2 we compare the master curve $D(t)$ of the film with the master curve $J(t)$ of bulk PS of high MW=600,000 Da, which has not been previously published. The slope of the log$\log$ plots of the data in the sub-Rouse regime of the thin film is about a factor of 2 smaller than that in the bulk. This significant change of slope in thin film can be taken as additional evidence of the sub-Rouse modes contributing to higher compliance are shifted to shorter tomes less than slower sub-Rouse modes contributing to lower compliance. These experimental findings had been explained $[6,14]$ by the Coupling Model (CM) Eqs. (1) and (2) from the segmental $\alpha$-relaxation coupling parameter $n_{\alpha}$ being larger than all the coupling parameters $n_{S R}$ of the sub-Rouse modes, and also the faster sub-Rouse mode contributing to larger value of $\widehat{D}_{S R}(t)$ has smaller coupling parameter $n_{s R}$. On decreasing the film thickness $h$, the coupling parameters $n_{\alpha}(h)$ and $n_{s R}(h)$ are reduced, but the enhancement of mobility is much larger for all the modes with larger coupling parameter [13]. Thus the faster sub-Rouse 
modes lag behind the slower sub-Rouse modes, and all of them lag behind the segmental $\alpha$ relaxation in their shifts to shorter times. This effect of bifurcation of the sub-Rouse modes from the segmental $\alpha$-relaxation is absent in thick films like bulk, but becomes increasingly important on decreasing $h$. When the master curves of creep compliance data constructed for different film thickness $h$ are presented and compared over the same time window (see Fig.9 in [9]), the effect shown is the decrease of the plateau rubbery compliance on decreasing $h$. Thus the simultaneous accelerations of the sub-Rouse modes and the segmental $\alpha$-relaxation but to a less degree for the former than the latter give an explanation of the decrease of the plateau rubbery compliance on thinning the film observed by McKenna and coworkers. Other details of the explanation were given before in [6]. Exactly the same as described in the above for PS was found in polycarbonate by McKenna and co-workers [2]. Their creep compliance data of the $22 \mathrm{~nm}$ film in Fig.2a [2] and the master curve in Fig.2b have essentially the same properties as Figs. 1 and 2 herein, from which we can reach the same conclusions.

There is an analogue of the effect found by McKenna et al. on reducing the film thickness of high MW PS. Instead of thinning the PS film, reduction of $n_{\alpha}$ and $n_{S R}$ in bulk PS can be achieved by dissolving PS in the solvent tri-m-tolyl phosphate [34]. The presence of the solvent increases the average separation the repeat units to mitigate the intermolecular interaction, and hence reduce the coupling parameters of all modes. Creep compliance measurements of $17 \%$ polystyrene solution [34] have shown that the segmental $\alpha$-relaxation shifts to shorter times much more than the sub-Rouse modes, resulting in a much broader glass-rubber transition zone in the solution than in bulk PS. The retardation spectra of the bulk PS and its 17\% solution in Fig.1 of [34] clearly demonstrate the acceleration by dissolution of the segmental $\alpha$-retardation times by reduction of $n_{\alpha}$ is larger than the sub-Rouse modes by 
reduction of $n_{S R}$. Moreover observed is a change in the shape of the retardation spectrum indicating the sub-Rouse mode with longer retardation time is shifted to shorter times more than the sub-Rouse mode with shorter retardation time. This effect is the same found by McKenna and coworkers in the change of the creep compliance on reducing the thickness of the PS thin films, and the CM explanation [6] given applies to the retardation spectra of the $17 \%$ polystyrene solution as well.

There is evidence of the sub-Rouse modes from the center-of-mass MSD data from neutron spin echo experiments and the theoretical predictions from Guenza's theory of cooperative dynamics from generalized Langevin equation for samples with number of segments $N=36,106,192$, and 377 of the protonated diffusion polyethylene chains [23,24]. The Rouse modes for short chains obey Brownian diffusion and has MSD proprotional to $t^{1.0}$. Experimental data as well as theory show $t^{1.0}$-dependence of MSD at long times, but subdiffusive behavior at shorter times, which corresponds to the sub-Rouse modes.

\section{The Sub-Rouse Modes Causing the Upper Glass Transition and Responding to Physical Aging}

As shown in Figs.1 and 2, the sub-Rouse modes had also been seen directly in creep compliance measurements on nanobubble-inflated freestanding polymer thin films. On decreasing film thickness, the sub-Rouse modes shift to shorter times like the segmental $\alpha$ relaxation, but the shift of the former is less than the latter $[1,2,6]$. The resulting effects are the increasing separation of the sub-Rouse modes from the segmental $\alpha$-relaxation, and the observed decrease of the rubbery plateau, which had been explained by the Coupling Model as originating from the lesser enhancement of mobility of the sub-Rouse modes than the 
segmental $\alpha$-relaxation in thin films [6]. These effects lead naturally to the existence of two transitions found by ellipsometry in freestanding high molecular weight polystyrene films. The upper transition at a higher temperature, $T_{g}^{u}$, originates from the sub-Rouse modes, and the lower transition at $T_{g}^{l}$ is from the segmental $\alpha$-relaxation.

In this paper we present new materials to demonstrate from experimental evidences that the sub-Rouse modes are coupled to density in a manner similar to the segmental $\alpha$ relaxation. One consequence of this inherent property of the sub-Rouse modes is that they can cause the upper glass transition. Another consequence is the shift of the modes to longer times on physical aging polystyrene, in the bulk or in thin films, which proceeds simultaneously with the densification of the material with increase of aging time. To support this, let us examine some actual physical aging experimental data of bulk polymers obtained by creep compliance and stress relaxation. After rapid cooling of a PS with $M_{w}=3.8 \times 10^{6} \mathrm{~g} / \mathrm{mol}$ from $100^{\circ} \mathrm{C}$ down to $90^{\circ} \mathrm{C}$, Plazek and coworkers [35] measured the creep compliance, $J(t)$, at several stages after aging times $t_{a g e}=10,56,230$, and $7230 \mathrm{~min}$. The data with $J(t)<10^{-9.6}$ $\mathrm{cm}^{2} /$ dyne are shown by symbols in Fig. 3 The longest measurement time of $J(t)$ at each stage is much less than $t_{\text {age }}$ to ensure the measured $J(t)$ is for the structure at $t_{\text {age }}$. This is the reason why only the segmental $\alpha$-relaxation and none of the sub-Rouse modes contributing to $J(t)$ at higher values with longer retardation times were obtained. Nevertheless, the value of $J_{e \alpha} \approx$ $4 \times 10^{-9} \mathrm{~Pa}^{-1}$ is shown by the broken line. Approximately values of $J(t)$ below and above this demarcation line are contributed by the segmental $\alpha$-relaxation and sub-Rouse modes respectively. The red line represents $J(t)$ obtained at temperature above $T_{g}$ of the same polymer at equilibrium. As expected, the $J(t)$ obtained at equilibrium does not describe well the data of the rapidly quenched sample after $t_{a g e}=10 \mathrm{~min}$. Notwithstanding, the $J(t)$ curve is 
drawn as a reminder of the presence of the sub-Rouse modes at any stage of aging. The black curve is obtained by shifting the red curve horizontally until there is agreement with the data after aging up to $t_{a g e}=7230 \mathrm{~min}$. Better agreement of the black curve with the data (black squares) is because after 5 days of aging, equilibrium is nearly attained. The part of the black curve with $J(t)>J_{e \alpha} \approx 4 \times 10^{-9} \mathrm{~Pa}^{-1}$ is not included for reason which will become clear below.

By inspection of the data in Fig.3, the shift of the segmental $\alpha$-relaxation data to longer times with increase of aging time is clear and as expected. If the notion that the subRouse modes do not change at all were correct, then the contribution to $J(t)$ of the sub-Rouse modes at $t_{\text {age }}=10$ min (represented by the black filled squares on top of the red curve in Fig.3) would not shift on increase of $t_{\text {age }}$. The consequence is that at the last stage of aging with $t_{\text {age }}=7230 \mathrm{~min}, J(t)$ will increase approximately as indicated by the black curve up to $J_{e \alpha} \approx$ $4 \times 10^{-9} \mathrm{~Pa}^{-1}$, but no further. This is because the sub-Rouse part contributing to $J(t)>J_{e \alpha}$ (black squares on top of red line) does not shift to longer times according to the notion of the sub-Rouse modes are insensitive to physical aging. The consequence is a untenable change of viscoelastic properties of PS on aging, i.e. the compliance of the aged polymer becomes discontinuous at the compliance level of $J_{e \alpha} \approx 4 \times 10^{-9} \mathrm{~Pa}^{-1}$. The discontinity implies that the aged polymer is no longer behaving as a polymer anymore on aging, which is certainly not true. Therefore, the sub-Rouse modes of bulk PS in the non-equilibrium glassy state do shift to longer times on aging, indicating that the sub-Rouse modes are coupled to density, and their relaxation times increase in response to density change and vice versa.

Albeit weaker than the Vogel-Fulcher-Tammann-Hesse (VFTH) dependence of $\tau_{\alpha}[15]$ the temperature dependence of relaxation times of $\tau_{s R}$ is another VFTH-dependence in bulk 
polymers $[17,18,22,36]$. Since the VFTH-dependence of relaxation time originates from sensitivity to change in density or entropy on cooling, this fact also indicates the sub-Rouse modes are coupled to density. On physical aging, the approach of the density or the fictive temperature of the glass towards equilibrium value is effected by all viscoelastic mechanisms that are coupled to density, and that include not only the segmental $\alpha$-relaxation, but also the sub-Rouse modes.

Actually photon correlation spectroscopy (PCS) measurements [37] have found the sub-Rouse modes in several polymers even before the creep compliance measurements [30], but it was not called as such. These chain modes were found in 1984 by Fytas et al. [37] in low molecular weight (MW) poly(methylphenylsiloxane) (PMPS) with MW=2500 g/mol at different pressures from 1 to 1750 bar and temperatures between 269 and $308 \mathrm{~K}$. The measured time correlation function show a faster segmental $\alpha$-relaxation and a slower $q^{2}$ dependent chain diffusional process due to local concentration fluctuations. The latter is actually the sub-Rouse modes in this low MW PMPS, but not recognized as such in this early study, and also in another study of PMPS with MW=5000 g/mol ten years later in 1994 by the combination of creep compliance and PCS [17]. Observed in the 1984 paper is that the mean characteristic times of the two processes approach each other at low temperatures and high pressures, which is the behavior exhibited by the sub-Rouse modes and the segmental $\alpha$ relaxation as we now know in several polymers. The explanation of this thermorheological complex behavior was given two years later in 1986 [38], and the explanation is based on $n_{s R}$ being smaller than $n_{\alpha}$, consistent with papers published in the the next decade $[6,22,28,31$, 33, 36]. The creep compliance data [17] of PMPS with MW=5000 g/mol enable the 
determination of the range of compliance contributed by the segmental $\alpha$-relaxation, which is given by

$$
J_{g}=5.39 \times 10^{-10} \mathrm{~Pa}^{-1} \leq \hat{J}_{\alpha}(t) \leq J_{e \alpha} \approx 2.82 \times 10^{-9} \mathrm{~Pa}^{-1}
$$

Hence the data observed at compliance higher than $J_{e \alpha} \approx 2.82 \times 10^{-9} \mathrm{~Pa}^{-1}$ come from the sub-Rouse modes. The ratio, $J_{e \alpha} / J_{g} \approx 5.2$, of PMPS is comparable to that of PS given by Eq.(1), in support that the size of the contribution to compliance from the segmental $\alpha$ relaxation is about the same size for polymers in general. By the way, the contribution to $J_{e \alpha} / J_{g}$ from the $\alpha$-relaxation in non-polymeric glass-formers such as tri-naphthyl benzene [39] is about 2.5 and smaller than polymers. The sub-Rouse modes were even more clearly resolved from the segmental $\alpha$-relaxation in poly(methyl-para-tolyl-siloxane) (PMpTS) of MW=4500 and 42,000 g/mol by PCS experiments and shear modulus measurements [40]. The pressure dependencies of chain modes in unentangled to entangled polyisoprene was found by Floudas and coworkers [41-43].

The fact that the relaxation time $\tau_{s R}$ of the sub-Rouse modes of PMPS is pressure dependent $[17,37]$ and are coupled to density was confirmed in details by a more recent study of the dynamics of PMPS with $\mathrm{MW}=10500 \mathrm{~g} / \mathrm{mol}$ and PDI=1.04 by photon correlation spectrscopy (PCS) under applied pressure from 1 to 900 bars and temperature in the range from 247-278 K by Kriegs et al.[46]. The $\alpha^{\prime}$ mode in the PCS is the sub-Rouse modes like in [17]. These authors found both the relaxation times of the segmental $\alpha$-relaxation and the subRouse modes are temperature and pressure dependent, and well described by two different VFTH laws like in other polymers [21], and the two different forms of $\langle\tau\rangle=\tau_{P} \exp \left[D_{P} P /\right.$ $\left.\left(P_{0}-P\right)\right]$. From the pressure dependencies, the activation volumes for both processes are very similar, and both relaxation times, $\tau_{s R}$ and $\tau_{\alpha}$, are functions of the combined variable, 
$T / \rho^{\gamma}$, with the same $\gamma$ where $\rho$ is the density, like that found in other polymers including polyisoprene, polypropylene glocol, and polyoxybutylene by dielectric relaxation [46-48]. This experiment shows beyond any doubt that the sub-Rouse modes are coupled to density like the segmental $\alpha$-relaxation. Hence if physical aging is performed at temperature below the upper glass transition temperature $T_{g}^{u}(h)$ of the freestanding film [3], $\tau_{s R}$ of the sub-Rouse modes increases and concomitantly the density also increases due to their coupling.

\section{Sub-Rouse modes are necessary to explain surface viscosity measurements}

A novel investigation of enhanced surface mobility was reported by Chai et al. [4], using the geometry of a stepped PS film on a substrate. They measured the viscosity above and below the bulk $T_{g B}$ of the low molecular weight PS with $M_{\mathrm{w}}=3000 \mathrm{~g} / \mathrm{mol}$. At temperatures above the bulk $T_{g B}=70 \mathrm{C}$, the entire film is involved in viscous flow. However, below $T_{g B}$, flow occurs only in the near-surface region, made possible by the high mobility at the surface. At temperatures sufficiently far below $T_{g B}$, the flow measured by Chai et al. comes totally at near the surface.

Before we proceed further in considering the surface viscosity data of Chai et al.[4] and similar ones of Yang et al. [5], it is important to recognize from creep compliance measurements of low molecular weight PS that present are the segmental $\alpha$-relaxation and the sub-Rouse modes, but not the Rouse modes. This is because the chains are too short to support compliance contributed by the Rouse modes determined by Eq.(5). The shear compliance data of Plazek and O'Rourke [15] for PS with low MW=3400 g/mol and $T_{g} \approx 70$ ${ }^{\circ} \mathrm{C}$ are shown in Fig.4, where the horizontal lines indicate approximately the compliance contributed by the sub-Rouse modes. From about $100{ }^{\circ} \mathrm{C}$ down to $T_{g} \approx 70{ }^{\circ} \mathrm{C}$, they found the 
recoverable compliance, $J_{r}(t)=J(t)-t / \eta$, are all less than $J_{S R} \approx 10^{-7} \mathrm{~Pa}^{-1}$, and hence the data are contributed only by the sub-Rouse modes and the segmental $\alpha$-relaxation. The final increase of $J_{r}(t)$ is due to the presence of a higher molecular weight tail in the polydisperse sample. The characteristics of the $3000 \mathrm{~g} / \mathrm{mol}$ low molecular weight PS studied by Chai et al. are close to that of Plazek and O'Rourke including the same bulk $T_{g B}=70{ }^{\circ} \mathrm{C}$. Therefore, the creep data in Fig.3 is applicable to the study by Chai et al. The sample of Yang et al. with $\mathrm{MW}=2400 \mathrm{~g} / \mathrm{mol}$ and $T_{g B}=64{ }^{\circ} \mathrm{C}$ is not too different either. At temperatures above and below the bulk $T_{g B}$ in the two surface viscosity studies, the only viscoelastic mechanisms present are the segmental $\alpha$-relaxation and the sub-Rouse modes. Viscous flow of polymers is carried out exclusively by the polymer chain modes, and not by the segmental $\alpha$-relaxation. In the present cases, the polymers are the low molecular weight PS, and the chain modes are specifically the sub-Rouse modes. Consequently the sub-Rouse modes are the relevant viscoelastic mechanism to consider in explaining the data of Chai et al. and Yang et al. in the bulk and at the surface. Works by others [3-5] in the past had not considered the sub-Rouse modes in the bulk and how they are modified at the surface or in thin films of PS. Thus the presentations in the previous sections are relevant as well as essential to show the sub-Rouse modes are shifted to shorter times at the surface and in thin films of PS, and there is an explanation from the CM. Conversely, the enhancement of fluidity found experimentally $[4,5]$ is a direct proof that the sub-Rouse modes are shifted to shorter times at the surface, consistent with observation by creep compliance measurements of high MW thin PS films by McKenna et al. [1, 2].

Chai et al. was able to infer from the data that the surface viscosity is more than 3 orders of magnitude lower than the bulk viscosity at $T_{g B}$, and has the Arrhenius temperature dependence with activation energy $E_{a} \sim 337 \pm 20 \mathrm{~kJ} \mathrm{~mol}^{-1}$. Yang et al. measured the viscosity 
of the PS films on silicon at different temperatures and found that the transition temperature for the viscosity decreases with decreasing film thickness. By analyzing the data, they deduced the presence of a highly mobile surface liquid layer, which dominates the flow in the thinnest films studied, and has Arrhenius $T$-dependence with activation energy of $185 \mathrm{~kJ} / \mathrm{mol}$, consistent with the value of $230 \mathrm{~kJ} / \mathrm{mol}$ measured by lateral force microscopy and interpreted in terms of the CM by Tanaka et al. [49], and $150 \mathrm{~kJ} / \mathrm{mol}$ from other surface mobility measurements $[50,51]$.

The isothermal creep compliances data of the 3400 g/mol PS in Fig.4 show the steady state compliance, $J_{e}$, decreases on decreasing temperature [15]. From the Maxwell relation, $\tau_{s R}(T)=\eta(T) J_{e}(T)$, the sub-Rouse retardation times, $\tau_{s R}$, are determined from the known values of $\eta(T)$ and $J_{e}(T)$ given in [15]. The results are shown in the inset together with the segmental $\alpha$-relaxation times, $\tau_{\alpha}$. Measurements by photon correlation spectroscopy had determined the segmental $\alpha$-relaxation coupling parameter, $n_{\alpha}$, to have the value of 0.50 [52]. The weaker temperature dependence of $\tau_{s R}$ compared with $\tau_{\alpha}$ can be accounted for by Eqs.(1) and (2) the CM, and from which we estimate the value of the sub-Rouse modes coupling parameter, $n_{s R}$, to lying within the range, $0.20 \leq n_{s R} \leq 0.30$. As mentioned before, the 3400 $\mathrm{g} / \mathrm{mol}$ PS sample can be considered the same as the sample of Chai et al., and hence their result, that the surface viscosity is more than 3 orders of magnitude lower than the bulk viscosity at $T_{g B}=70 \mathrm{C}$, is equivalent to the reduction of $\tau_{s R}$ of the same magnitude. This is illustrated in Fig.5 by the downward pointing blue arrow at 1000/ $T_{g B}$ in the Arrhenius plot of $\tau_{s R}$ and $\tau_{\alpha}$. The top end of the arrow is located at 3 orders of magnitude lower than $\tau_{s R}\left(T_{g B}=70\right.$ C). The maximum reduction of sub-Rouse relaxation time possible at the surface is $\tau_{0, \mathrm{sR}}$, which can be obtained from the CM Eq.(2) with $0.20 \leq n_{s R} \leq 0.30$. The values of $\tau_{0, s \mathrm{R}}$ 
calculated within the bounds of $n_{s R}$ are shown by the purple vertical bar placed slightly to the right of 1000/T $T_{g B}$ in Fig.5. These estimates are consistent with the results stated by Chai et al. based on their own measurements.

There is no way to convert exactly the viscosity data in the bulk and at the surface of the $2400 \mathrm{~g} / \mathrm{mol}$ PS measured by Yang et al. because creep data are not available and the temperature dependent values of $J_{e}$ are not known. In view of the inadequacy, we use the data of the $3400 \mathrm{~g} / \mathrm{mol}$ PS just as a guide. We assume the bulk $\tau_{s R}$ at $T_{g B}=64{ }^{\circ} \mathrm{C}$ of the $2400 \mathrm{~g} / \mathrm{mol}$ PS has the same value as the bulk $\tau_{s R}$ at $T_{g B}=70{ }^{\circ} \mathrm{C}$ of the $3400 \mathrm{~g} / \mathrm{mol} \mathrm{PS}$, i.e. the ratio of $\tau_{s R} / \tau_{\alpha}$ at $T_{g B}=64{ }^{\circ} \mathrm{C}$ for the $2400 \mathrm{~g} / \mathrm{mol}$ sample is the same as that at $T_{g B}=70{ }^{\circ} \mathrm{C}$ for the 3400 $\mathrm{g} / \mathrm{mol}$ sample. With this assumption, both the logarithm of the bulk viscosity and surface viscosity of the thinnest $2.3 \mathrm{~nm}$ film data of Yang et al. are shifted vertically by one and the same constant value to obtain the bulk and surface $\tau_{s R}\left(T_{g B}=64{ }^{\circ} \mathrm{C}\right)$ of the $2400 \mathrm{~g} / \mathrm{mol}$, as shown by the larger filled red and open circles respectively in Fig.5. Moreover, assuming the values of the bulk sub-Rouse coupling parameter $n_{s R}$ is the same as the $3400 \mathrm{~g} / \mathrm{mol}$ PS, i.e. $0.20 \leq n_{s R} \leq 0.30$, the calculated values of $\tau_{0, \mathrm{sR}}$ are bounded within the limits of the vertical bar, which are consistent with the result of Yang et al. represented by the large open circle in Fig.5.

To emphasize the orders of magnitude less enhancement of mobility of sub-Rouse modes compared to segmental $\alpha$-relaxation, the primitive segmental $\alpha$-relaxation time, $\tau_{0, \alpha}$, of the $3400 \mathrm{~g} / \mathrm{mol}$ PS is calculated by Eq.(1) with $n_{\alpha}=0.50$ at $T_{g B}=70 \mathrm{C}$. The result is represented by the blue diamond in Fig.5. This estimate of the maximum reduction of bulk $\tau_{\alpha}$ by the calculated $\tau_{0, \alpha}$ has been successful in accounting for ultrathin high MW freestanding film [14] 
where the surface is considered most instrumental [11-13] in reducing $T_{g}$, and by the lateral force spectroscopy on surface of PS [49].

Large enhanced surface diffusion has been observed in indomethacin, a small molecular glass-former [53]. It is also found in the surface of shear bands of mechanically deformed metallic glasses [54]. In both cases, the Coupling Model (CM) is able to explain [55.56] quantitatively the large enhancement of mobility at the surface from experiments.

There is no doubt that the free surface is an important cause of enhanced mobility of both the segmental $\alpha$-relaxation and the sub-Rouse modes of polymers. The latter is amply demonstrated by the surface flow experimental data [4,5] considered in this work. Notwithstanding, finite size effect is another contributing factor in polymer thin films when the thickness is comparable to the cooperative length-scale of the segmental $\alpha$-relaxation. It acts alone in causing significant reduction of $T_{g}$ in systems without free surface as shown by experiments [57-59]. Notable examples include the confinement of PMPS in nanocomposites [57], in nanometer glass pores of PDMS and PMPS by Schonhals and coworkers [58], and the study by Simon and coworkers [59].

\section{Conclusions}

Previous biaxial creep compliance measurements of PS and PC thin films of high molecular weight by McKenna and coworkers $[1,2]$ have already shown evidence of enhancement of the mobility of the sub-Rouse modes. This finding is pivotal in stimulating our attempts $[6,14]$ to explain the novel dynamics at free surface and in freestanding thin films of polystyrene [1-5] considered in this perspective. Most recent creep compliance measurements of thin films of a variety of polymers $[60,61]$ are consistent with the trend predicted [6]. These advances lend 
further support of the difference between the sub-Rouse modes and the segmental $\alpha$ relaxation in the enhancement of mobility at free surface and in freestanding films, which is basis of our explanation of the novel dynamics [6, 14]. It is important to note that the differences in dynamics between the segmental $\alpha$-relaxation, the sub-Rouse modes, and the Rouse modes were present in the bulk polymers and discovered by Plazek [15-17], and confirmed by others [18]. Breakdowns of thermo-rheological, piezeo-rheological, and $T \rho^{-\gamma}{ }_{-}$ rheological simplicities have been found in bulk polymers [32]. Restated as breakdown of thickness-rheological simplicity, it become obvious that the findings of McKenna and coworkers in thin polymer films are akin to the breakdown of thermo-rheological simplicity. Naturally, viable explanation of the polymer thin films data is expected to be related to the explanation of the viscoelastic anomaly of bulk polymers.

In this perspective, we provide previously unpublished prediction on the effect of physical aging of freestanding PS films, and explanation of the surface viscous flow experiment [4,5]. Supported by creep compliance, dielectric and photon correlation spectroscopy measurement of low molecular weight PS and other polymers, we show that the sub-Rouse modes are cooperative, coupled to density, and responsible for the upper glass transition of high molecular weight freestanding PS films. The sub-Rouse relaxation times will increase on physical aging, and brings along an increase in density due to the coupling. No doubt the sub-Rouse modes are responsible for viscous flow in the bulk and at the free surface of low molecular weight PS. The experimental observed large reduction of viscosity at the free surface is direct evidence of enhancement of mobility of the sub-Rouse modes by the mitigation of intermolecular coupling at the surface, occurring simultaneously with the same effect on the segmental $\alpha$-relaxation. Notwithstanding, at the surface, the reduction of 
viscosity from the sub-Rouse modes is significantly smaller than that of the segmental relaxation time. It is worthwhile to point out that in publishing the measurement of viscosity of low molecular weight polystyrene at the surface $[4,5]$ the authors made no distinction between segmental $\alpha$-relaxation and the sub-Rouse modes in their papers. This is a shortcoming which could be avoided if attention were paid to advances in viscoelastic properties of bulk polymers [16,18,22,28-38].

Altogether, the recent advances in the study of dynamics of polymer thin films and at the free surface have shown not only the change of the glass transition temperature effected by the segmental $\alpha$-relaxation is interesting, but also that of the sub-Rouse modes. The Coupling Model had been successful in accounting for the viscoelastic anomalies caused by the breakdown of thermo-rheological complexity, piezo-rheological complexity, and $T \rho^{-\gamma}-$ rheological complexity of bulk polymers [32]. It continues to explain the differences in the changes of the segmental $\alpha$-relaxation and the sub-Rouse modes at the surface and in thin films of high and low MW PS.

\section{References}

[1] P. A. O’Connell, S. A. Hutcheson, and G. B. McKenna, J. Polym. Sci., Part B: Polym.

Phys. 46, (2008) p.1952.

[2] P. A. O’Connell, J. Wang, T. Adeniyi Ishola, and G. B. McKenna, Macromolecules 45, (2012) p. 2453.

[3] J. E. Pye and C. B. Roth, Phys. Rev. Lett. 107 (2011) p.235701.

[4] Y. Chai, T. Salez, J. D. McGraw, M. Benzaquen, K. Dalnoki-Veress, E. Raphaël, J. A. Forrest, Science, 343 (2014) p. 994. 
[5] Z. Yang, Y. Fujii, F. K. Lee, C-H Lam, and O. K. C. Tsui, Science 328 (2010) p. 1676.

[6] K. L. Ngai, Daniele Prevosto, and Luigi Grassia, J. Polymer Sci. B: Polym. Phys. 51 (2013) p.214.

[7] K. Dalnoki-Veress, J. A. Forrest, C. Murray, C. Gigault, and J. R. Dutcher, Phys. Rev. E 63 (2001) p.031801.

[8] J. Mattsson, J. A. Forrest, and L. Borjesson, Phys. Rev. E 62 (2000) p.5187.

[9] C. Rotella, S. Napolitano, and M. Wubbenhorst, Macromolecules 42 (2009) p. 1415.

[10] S. Napolitano, and M. Wübbenhorst, Polymer 51 (2010) p. 5309.

[11] K. L. Ngai, Eur. Phys. J. E 8, 225 (2002); Philos. Mag. B 82 (2002) p.291.

[12] K. L. Ngai, Eur. Phys. J. E 12 (2003) p.93.

[13] K. L. Ngai, J. Polym. Sci.,Part B: Polym. Phys. 44 (2006) p.2980.

[14] D. Prevosto, S. Capaccioli, and K.L. Ngai, J. Chem.Phys. 140 (2014) p.074903.

[15] D. J. Plazek, M. J. O’Rourke, M. J. Polym. Sci. Polym. Phys. Ed. 9 (1971) p.209.

[16] D.J. Plazek, J. Rheology 40 (1996) p.987.

[17] D.J. Plazek, C. Bero, S. Neumeister, G. Floudas, G. Fytas, and K.L. Ngai, J. Colloid Polymer Sci. 272 (1994) p.1430.

[18] K. L. Ngai and D. J. Plazek, Rubber Chem.Tech. Rubber Review 68 (1995) p.376.

[19] J. Y. Cavaille, C. Jordan, J. Perez, L. Monnerie, G. P. Johari, J. Polym. Sci.:Part B: Polym. Phys. 25 (1987) p.1235.

[20] A. Patkowski, J. Gapinski, T. Pakula, and G. Meier, Polymer 47 (2006) p. 7231.

[21] X. Wu, C. Liu, Z. Zhu, K.L. Ngai, and L.M. Wang, Macromolecules 44 (2011) p. 3605.

[22] K. L. Ngai, D. J. Plazek, and R. W. Rendell, Rheol. Acta 36 (1997) p.307.

[23] M. G. Guenza, Phys. Rev. E 89 (2014) p.0526031. 
[24] M. Zamponi, A. Wischnewski, M. Monkenbusch, I. Willner, D. Richter, P. Falus, B. Farago, B.; M.G. Guenza, J. Phys. Chem. B 112 (2008), p.1622.

[25] K.L. Ngai, Relaxation and Diffusion in Complex Systems, Springer, NY 2011.

[26] V.G. Sakai, J.K. Maranas, Z. Chowdhuri, I. Peral, J.R.D. Copley, J. Polym. Sci. B Polym. Phys. 43 (2005) p. 2914.

[27] J.L. Keddie, R.A.L. Jones, R.A. Cory, Europhys. Lett. 27 (1994) p.59.

[28] K. L. Ngai, A. Schonhals, and E. Schlosser, E. Macromolecules 25 (1992) p. 4915.

[29] D. J. Plazek, E. Schlosser, A. Schönhals, and K. L. Ngai, J. Chem. Phys. 98 (1993) p. 6488.

[30] D. J. Plazek, I. C. Chay, K. L. Ngai, and C. M. Roland, Macromolecules, 28 (1995) p.6432.

[31] M. Paluch, S. Pawlus, A.P. Sokolov, and K. L. Ngai, Macromolecules, 43 (2010) p. 3103.

[32] K. L. Ngai and D. J. Plazek, Macromolecules, 47 (2014) p.8056.

[33] X. Wu, C. S. Liu, and K. L. Ngai, Soft Matter, 10 (2014) p.9324.

[34] K. L. Ngai, D. J. Plazek, Macromolecules 35 (2002) p.9136.

[35] D.J. Plazek, K. L. Ngai, and R. W. Rendell, Polym. Engin. Sci. 24 (1984) p.1111.

[36] K. L. Ngai, D. J. Plazek, and A. K. Rizos, J. Polymer Sci. B: Polym.Phys. 35 (1997) p. 599.

[37] G. Fytas, Th. Dorfmuller, and B. Chu, J. Polym. Sci Polym. Phys. Ed, 22 (1984) p. 1471.

[38] K.L. Ngai and G. Fytas, J. Polym Sci. Polym Phys. Ed, 24 (1986) p.1683..

[39] D. J. Plazek and J. H. Magill, J. Chem. Phys. 45 (1966) p.3038.

[40] A. Patkowski, J. Gapinski, T. Pakula, G. Meier, Polymer, 47 (2006) p.7231. 
[41] G. Floudas, C. Gravalides, T. Reisinger, G. Wegner, J. Chem. Phys. 111 (1999) p. 9847.

[42] G. Floudas, Prog. Polym. Sci. 29 (2004) p.1143.

[43] G. Floudas, M. Paluch, A. Grzybowski, K. L. Ngai, K. L. Molecular Dynamics of GlassForming Systems: Effects of Pressure; Springer: Berlin, 2011.

[44] C. M. Roland, R. Casalini, M. Paluch, J. Polym. Sci. Polym. Phys. Ed. 42 (2004) p.4313.

[45] H. Kriegs, J. Gapinski, G. Meier, M. Paluch, S. Pawlus, and A. Patkowski, J. Chem. Phys. 124 (2006) p. 104901.

[46] C. M. Roland, M. Paluch, R. Casalini, J. Polym. Sci. B: Polym. Phys., 42 (2004) p. 4313.

[47] R. Casalini, and C. M. Roland, Macromolecules, 38 (2005) p. 1779.

[48] K.L. Ngai, R. Casalini, C.M. Roland, Macromolecules 38 (2005) p. 4363.

[49] K. Tanaka, A. Takahara, T. Kajiyama, Macromolecules 33 (2000) p.7588.

[50] Z. Fakhraai, J. A. Forrest, Science 319 (2008) p.600.

[51] R. M. Papaléo et al., Phys. Rev. B 74 (2006) p. 094203.

[52] A.K. Rizos and K.L. Ngai, Macromolecules 31 (1998) p. 6217.

[53] L. Zhu, C. W. Brian, S. F. Swallen, P. T. Straus, M. D. Ediger, and L. Yu, Phys. Rev. Lett. 106 (2011) p. 256103.

[54] J. Bokeloh, S. V. Divinski, G. Reglitz, and G. Wilde, Phys. Rev. Lett. 107 (2011) p. 235503.

[55] S. Capaccioli, K. L. Ngai, M. Paluch, and D. Prevosto, Phys. Rev. E 86 (2012) p. 051503.

[56] K. L. Ngai and Hai Bin Yu, J. Appl. Phys. 113 (2013) p.103508. 
[57] S.H. Anastasiadis, K. Karatasos, G. Vlachos, E. Manias, E.P. Giannelis, Phys. Rev. Lett. 84 (2000) p.915.

[58] A. Schönhals, H. Goering, Ch. Schick, B. Frick, R. Zorn, Eur. J. Phys. E Soft Matter 12 (2003) p.173; Colloid Polym. Sci. 282 (2004) p.882; J. Non-Cryst. Solids 351 (2005) p. 2668.

[59] Q. Li and S. L. Simon, Macromolecules, 42 (2009) p. 3573.

[60] M. Zhai and G. B. McKenna, Polymer 55 (2014) p.2725.

[61] X. Li and G. B. McKenna, Macromolecules, 48 (2015) pp 6329-6336. 


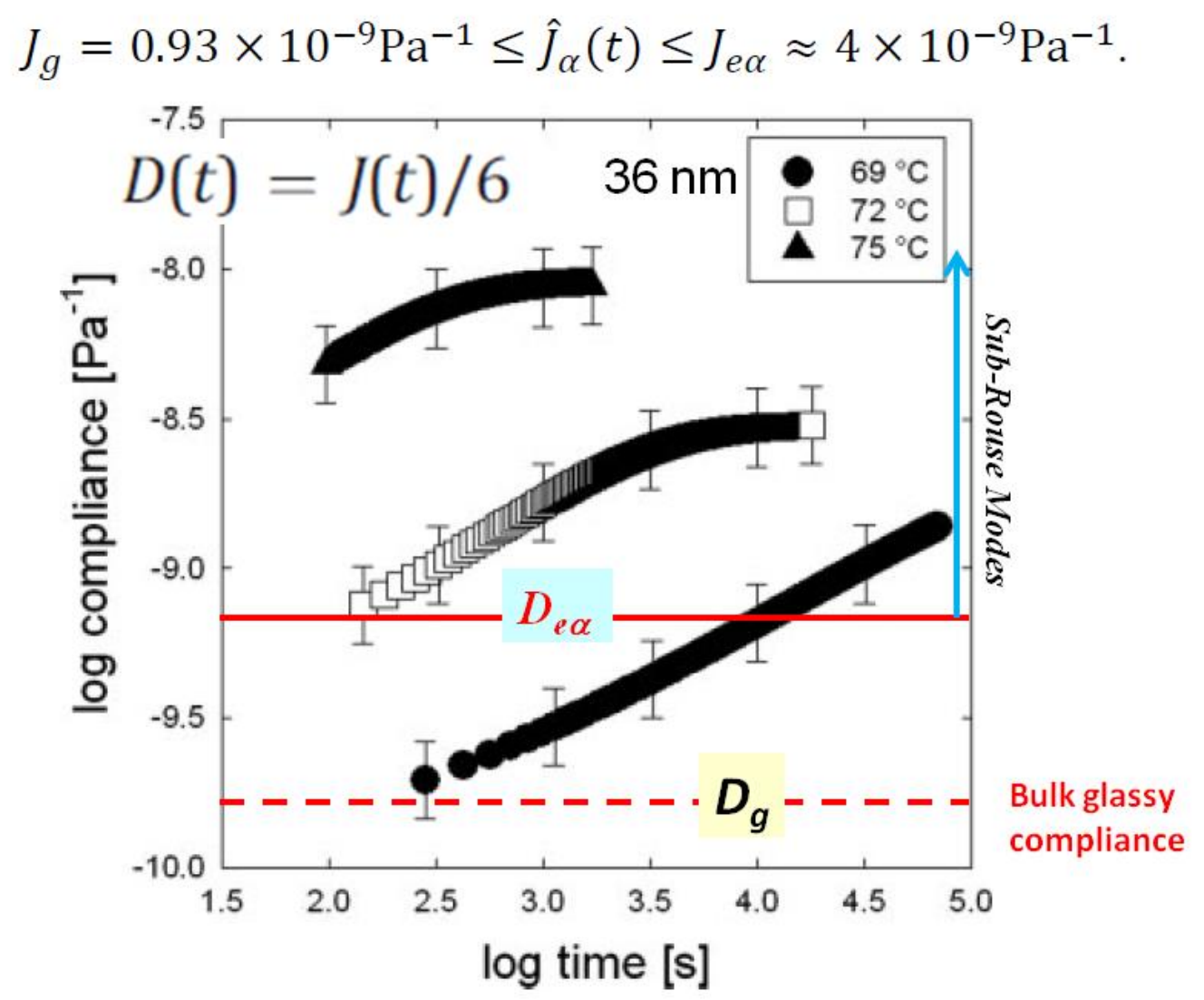

Fig.1. Log-log plot of creep compliance $J(t)$ as a function of time for a $36 \mathrm{~nm}$ thick PS film at temperatures of 69,72 , and $75.8^{\circ} \mathrm{C}$. Data from [1] redrawn. 

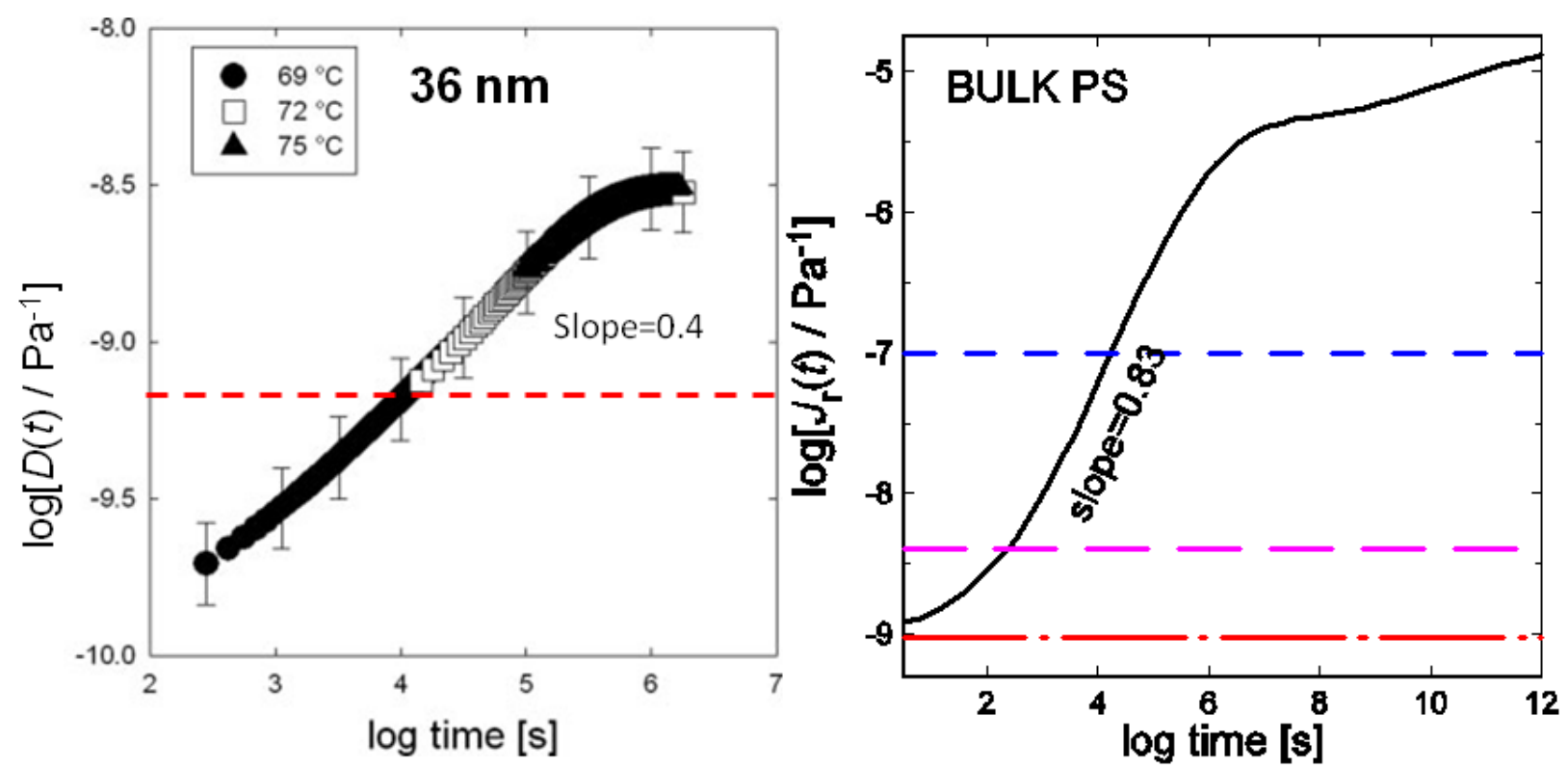

Fig, 2. (a) Master curve constructed from data $D(t)=J(t) / 6$ of the $36 \mathrm{~nm}$ thick PS film shown in Fig.1 (data from [1] redrawn). (b) Master curve constructed from the recoverable shear creep compliance $J_{r}(t)$ data of 600,000 Da bulk PS taken at temperatures above $T_{g}=100{ }^{\circ} \mathrm{C}$ by [15]. The horizontal lines show the bounds of the contributions from the segmental $\alpha$ relaxation and the sub-Rouse modes. 


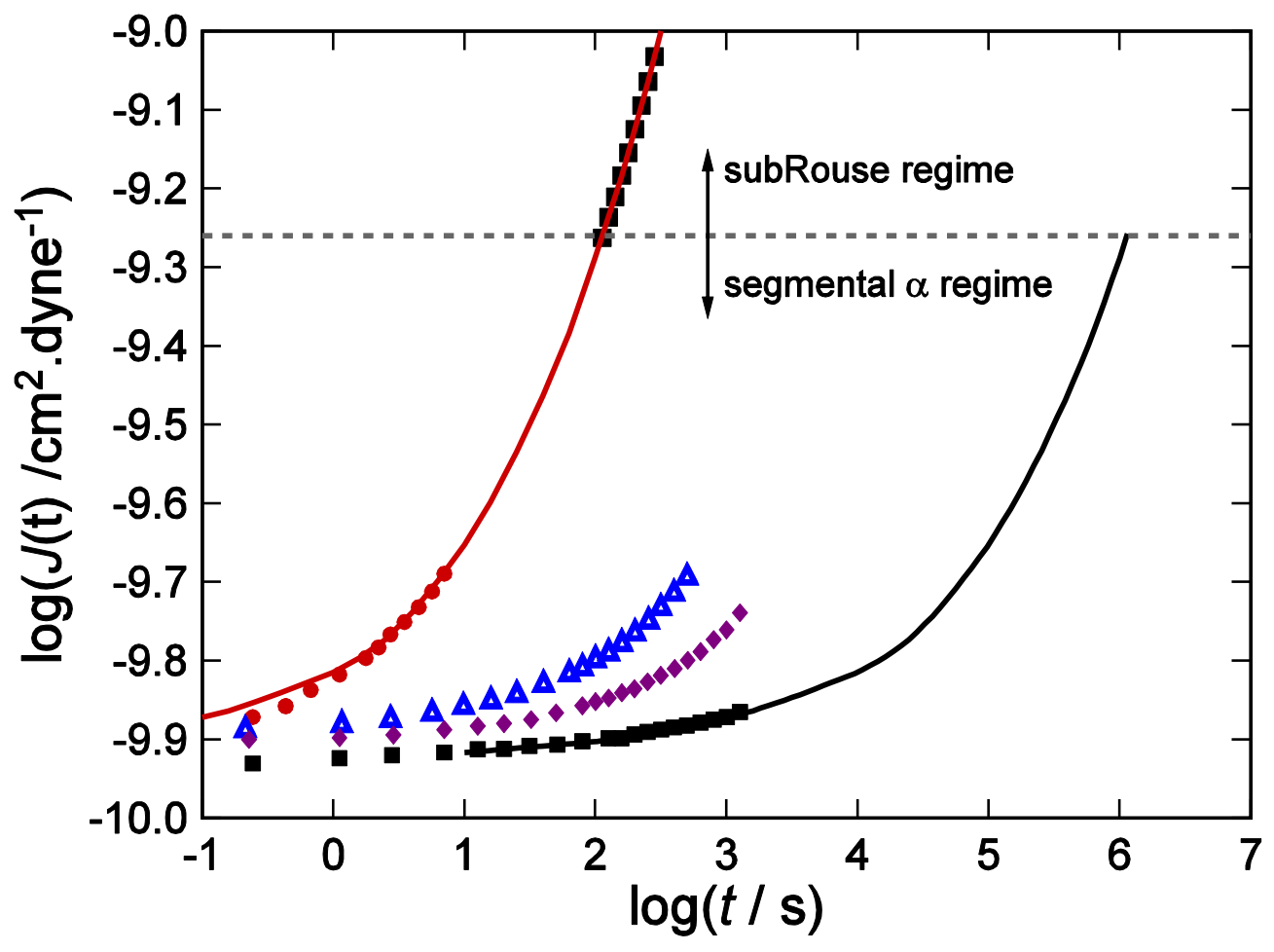

Fig.3. Selected creep compliance data, $J(t)$, represented by symbols were obtained at $90^{\circ} \mathrm{C}$ after cooling from $100^{\circ} \mathrm{C}$ with starting times (from left to right) of 10, 56, 230, and $7230 \mathrm{~min}$. Data taken from [35] and redrawn. For description of the lines and the black filled squares located above the broken line, see text. 


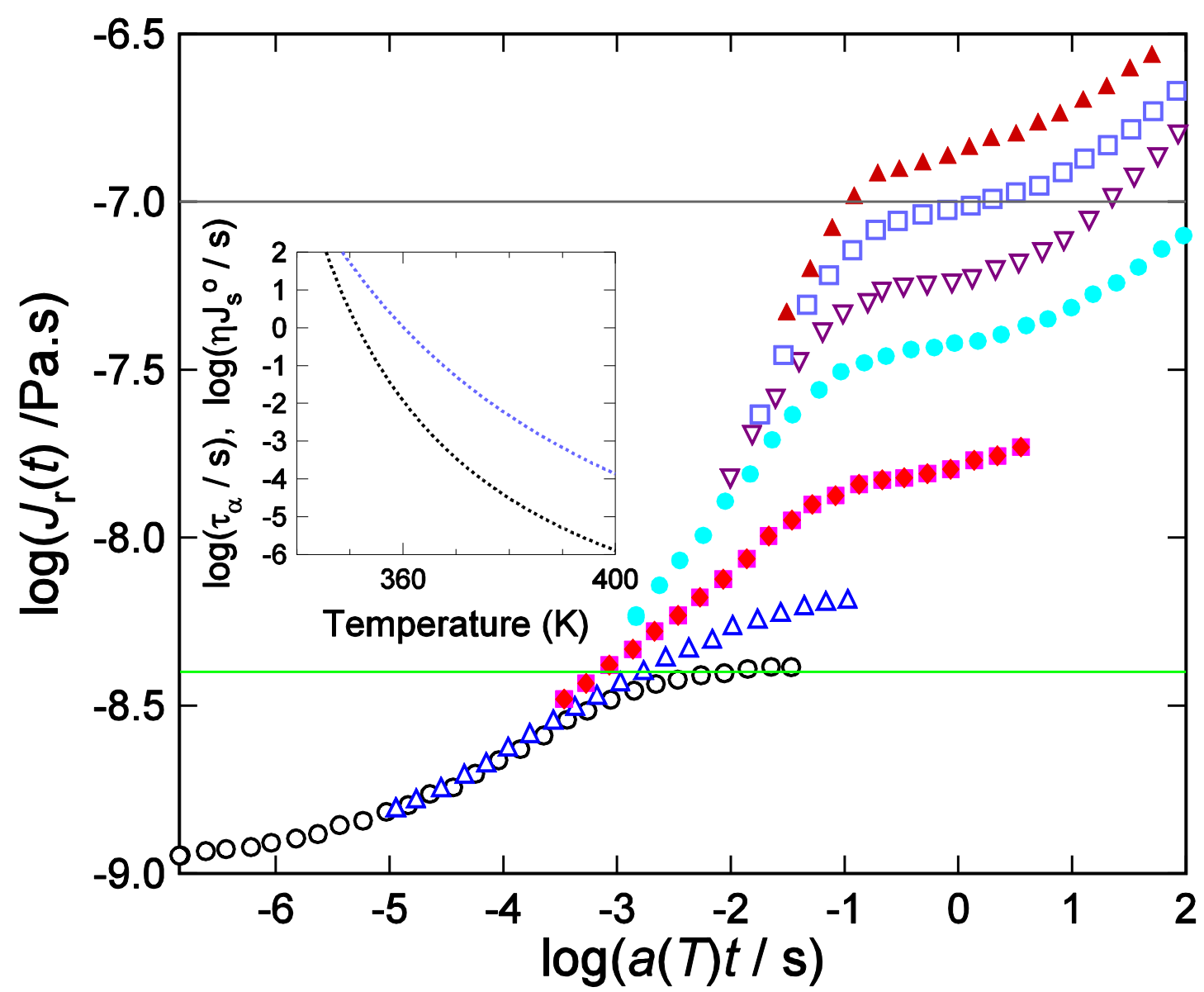

Fig, 4. Recoverable compliance for bulk PS with $\mathrm{MW}=3400 \mathrm{~g} / \mathrm{mol}$ reduced to $100^{\circ} \mathrm{C}$ by plotting logarithmically against the reduced time, t/a(T). Data from [17] are redrawn. Horizontal lines indicate the bounds of the contribution to compliance from the sub-Rouse modes. The inset shows the segmental realxation times $\tau_{s R}$ (black) and the sub-Rouse retardation times, $\tau_{s R}$ (blue). Data from [15] redrawn. 


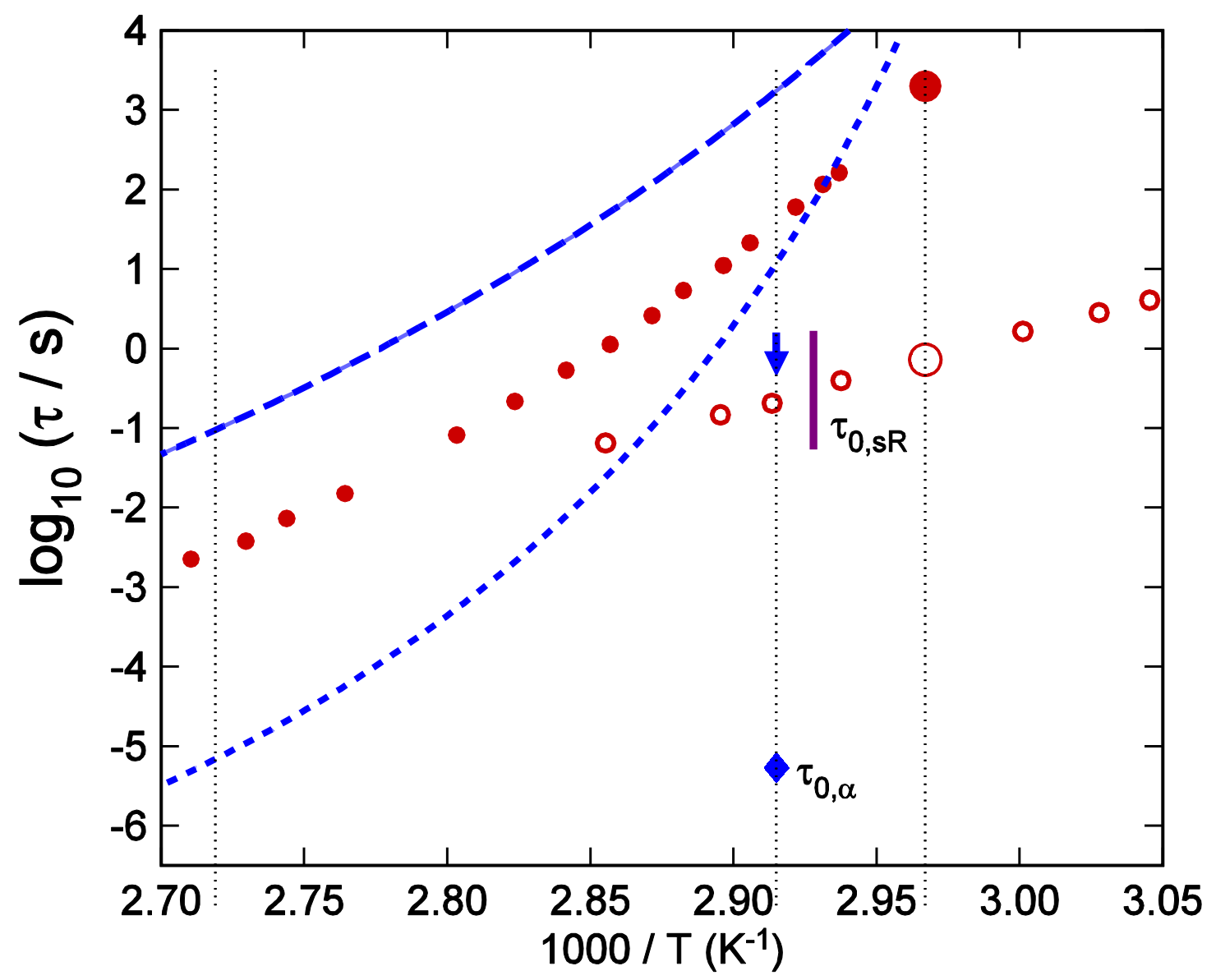

Fig. 5. The blue curves are $\tau_{\alpha}$ and $\tau_{s R}$ of bulk PS with MW=3400 g/mol taken from [15]. The filled and empty circles are the bulk and surface viscosity data of the $2400 \mathrm{~g} / \mathrm{mol}$ shifted in the manner as described in the text. The larger filled circle is $\tau_{s R}$ at $T_{g B}=64{ }^{\circ} \mathrm{C}$. For the larger open circle, the blue arrow and the vertical bar, see text. 